\title{
Limnological status and plankton dynamics in two fresh water wetlands of Aligarh, North India
}

\author{
Syed A. Untoo, *Saltanat Parveen and Uzma Ahmad \\ *Limnology Research Laboratory, Department of Zoology, Aligarh Muslim University, Aligarh. 202002 (U.P) \\ India; email: saltanatparveen@yahoo.co.in
}

\begin{abstract}
The present study deals with the plankton dynamics and physicochemical variables in two fresh water wetlands of Aligarh. Phytoplankton comprised five major groups, namely Myxophyceae, Chlorophyceae, Desmidiace, Bacillariophyceae and Euglenophyceae, while Zooplankton comprised four major groups, namely Cladocera, Rotifera, Copepoda and Ostracoda. Statistically, G.P.P. and N.P.P. values were found to have significant positive relationship with Chlorophyll ' $a$ ' and water temperature in both the wetlands, whereas the relationship with phytoplankton was observed non significant positive at Wetland $B$ and negative at Wetland $A$. Keywords: Wetlands, Phytoplankton, Zooplankton, G.P.P., N.P.P. , C.R. and Chlorophyll 'a'
\end{abstract}

\section{Introduction}

Wetlands are generally small in area, shallow and rain fed. They perform some useful functions in maintaining ecological balance of the nature (WWF, 1987). Wetlands show a wide spectrum of habitats ranging from extensive peat bogs of northern region to tropical mangrove forest; from seasonal ponds and marshes to flood plains and permanent riparian swamps; from fresh water ponds shallow lakes and large reservoirs to salt lakes, brackish lagoons, estuaries and coastal salt marshes.

The wetlands exhibit very large differences in their habitat characteristics such as hydrological regimes, bottom soil quality and in the nature and diversity of their biota. Wetlands provide people, directly or indirectly, with enormous range of goods and services like staple food, plants, fertile grazing land, and support for coastal and inland fisheries, flood control and breeding grounds for waterfowl, fish etc. Plentiful water and a high productivity have made wetlands among the richest and most diverse ecosystems of the world (WWF, 1992).

The plankton are small and microscopic organisms (Size: $<2$ micrometers - 200 micrometers) drifting or floating in the sea or fresh water, consisting chiefly of diatoms, protozoan, small crustaceans, and the eggs and larval stages of larger animals. Phytoplankton are the microscopic plants that act as the primary producers in an aquatic environment, convert acquired light energy into carbohydrates through photosynthesis. Energy not used by the phytoplankton for maintenance is available as food for the animals that consume it. Zooplankton are heterotrophic organisms that consume phytoplankton and others and in turn, they become food for larger, secondary consumer animals and fish.

The productivity in terms of planktonic biomass in fresh water lakes, rivers, ponds or wetlands, is regulated by various physico-chemical factors viz., temperature, transparency, $\mathrm{pH}$, electrical conductivity, hardness, nitrogen and phosphorus.

Unfortunately, rapid urbanization has led to the loss of wetland habitats through encroachment, bad management and pollution from sewage and waste and litter disposal activities. These factors have seriously affected the survival of these water bodies and posed serious threat to the flora and faunal supported by them.

The objective of the present study is to find out the limnological status, i.e., the water quality and plankton productivity in selected wetlands for their future utilization for fish culture.

Study Area:

\section{Material and Methods}

Aligarh city (North latitude $27^{\circ} 28^{\prime}$ and $28^{\circ} 10^{\prime}$ and East longitude $77^{\circ} 29^{\prime}$ and $78^{\circ} 36^{\prime}$ ) and its adjoining areas are richly well off with wetlands which support an extensive and regular fisheries of various kinds. They are surrounded by two river systems, Ganga and Jamuna with their many tributaries. In the present investigation two water bodies have been selected as wetlands to study their limnology, namely Wetland A and wetland B. Wetland A (WA) is locally termed as Dhobi-ghat, perennial, rectangular, eutrophic sewage fed wetland and used as drainage basin.

Wetland B (W B) is an old age water body having irregular shoreline and the main source of its water supply are the monsoon rains, village drainage and surface runoff from the adjoining areas. 


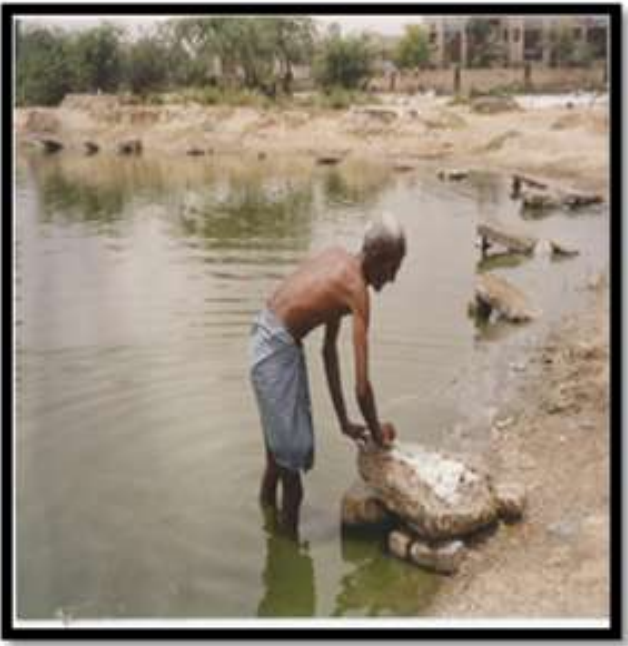

WA

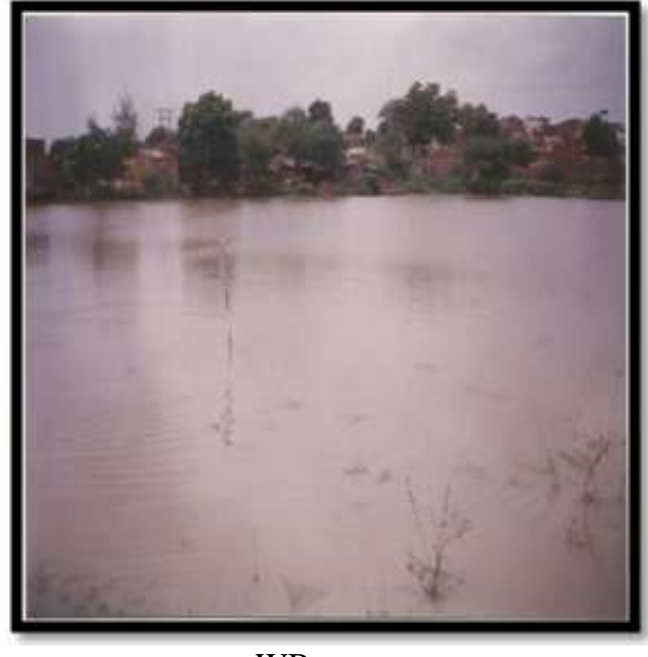

WB

Limnological Analysis:

Different physicochemical parameters were analyzed monthly from January 2000 to December 2001. Samples were collected from 8 am to $11 \mathrm{am}$. Air temperature and water temperature were recorded by mercury thermometer graduated upon $100^{\circ} \mathrm{C}$. Dissolved oxygen analysis was performed at the sites by Winkler's modified technique according to APHA (1998). $\mathrm{pH}$ was estimated by Digital $\mathrm{pH}$-meter. Turbidity was measured by Water Analyzer, Model no. WQC- 22A, Electrical conductivity was measured by conductivity meter. TDS was measured with the help of digital TDS meter.

For the estimation of Hardness, Chloride, Sulphate, Nitrate, Phosphate and Slilica, water sample was brought to the laboratory and analysis was done as per the standard methods given by APHA (1998) and Trivedi and Goel (1984).

Primary production was estimated by measuring the changes in dissolved oxygen concentration in light and dark bottles after following methodology of Gaarder and Gran (1927) and described by Strickland and Parsons (1972) and Vollenweider (1969). Chlorophyll 'a' was estimated after following methodology given by Trivedy and Goel (1984).

For Phytoplankton analysis, $500 \mathrm{ml}$ water sample was taken and treated with $5.0 \mathrm{ml}$ Lugol's solutions, qualitative and quantitative analysis were made of $20 \mathrm{ml}$ concentrate, which was obtained. The genera of phytoplankton were identified and enumerated following the works of Edmondson (1959), Needham and Needham (1962), Nayar et al. (1999) and Tonapi (1980).

For zooplankton analysis, samples were collected from each water body on a monthly basis. About 100 liters of water is filtered by passing water through plankton net made up of bolting silk cloth having mesh size of 25 micrometer. Samples were then washed into wide mouth bottles and were preserved by adding 5\% formaldehyde solution. Further analysis was done by putting $1 \mathrm{ml}$ of the preserved sample on a Sedgwick-Rafter cell and studying it under an inverted microscope. For qualitative analysis, the keys given in Edmondson (1959), Needham and Needham (1962), Pennak (1978), Tonapi (1980) and APHA (1998) were utilized and results were expressed in No./L.

\section{Statistical analysis:}

The correlation between various physico-chemical parameters of water samples were analyzed with the help of SPSS software (17.0) and Microsoft Excel.

\section{Results and Discussions}

In the studied wetlands the air temperature ranged from $17 \mathrm{C}^{\circ}$ to $36^{\circ} \mathrm{C}$, while water temperature from $14{ }^{\circ} \mathrm{C}$ to $32{ }^{\circ} \mathrm{C}$ (Table- 1), free $\mathrm{CO}_{2}$ was never recorded throughout the study period. The absence of $\mathrm{CO}_{2}$ is mainly due to its utilization during photosynthesis by algae or carbonates present did not allow the $\mathrm{CO}_{2}$ to be produced in the bottom and column to reach the surface (Ganapati, 1960). $\mathrm{pH}$ ranged from 8.3 to 9.2 during (Table 1). The variations in $\mathrm{pH}$ are linked with the chemical changes, species composition and life processes of animal and plant communities inhabiting the system.

Dissolved oxygen ranged from $4.0 \mathrm{mg} / \mathrm{l}$ to $9.6 \mathrm{mg} / \mathrm{l}$ (Table 2). Maximum values were recorded in June, 2001 and March 2001 in Wetland A. Higher values of dissolved oxygen during some months might be due to increased photosynthetic activity while lower values might be because of its utilization in decomposition of organic matter and respiration by micro and macro organisms. Total hardness is the concentration of divalent cations expresses as $\mathrm{CaCO}_{3}$. In most waters majority of cations are calcium and magnesium (Boyd, 1998). 
The values of total hardness were found to vary between $124.0 \mathrm{mg} / \mathrm{l}$ to highest $390 \mathrm{mg} / \mathrm{l}$ in these wetlands (Table 2). High values were, especially in Wetland A due to many stains, chemical, dyes and detergents are used by the washer man daily in these water bodies.

TDS values of Wetland B ranged from $600 \mathrm{mg} / \mathrm{l}$ to $5600 \mathrm{mg} / \mathrm{l}, 2$ (Table 2). High values may be due to more input of excess of allochthonous material. Chloride content of Wetland B varied from $50.0 \mathrm{mg} / \mathrm{l}$ to 318.0 $\mathrm{mg} / \mathrm{l}$. The concentration declined in the rains due to dilution of water. Sulphates content varied from $32.0 \mathrm{mg} / \mathrm{l}$ to $179.0 \mathrm{mg} / \mathrm{l}$ (Table 3 ). The higher concentrations of sulphates recorded in the study during summer months may be attributed to fast blowing hot and dry winds causing increased evaporations.

Phosphates in the Wetlands $0.191 \mathrm{mg} / \mathrm{l}$ to $1.425 \mathrm{mg} / \mathrm{l}$ in May, 200,1 whereas Nitrates ranged from 0.052 $\mathrm{mg} / \mathrm{l}$ to $0.278 \mathrm{mg} / \mathrm{l}$. Increased values during summer were mainly due to regeneration of inorganic phosphorous from the organic form during decomposition..

Silicates of Wetland B ranged from $0.0137 \mathrm{mg} / 1$ to $0.1887 \mathrm{mg} / \mathrm{l}$ in and in Wetland A it varied from $0.0218 \mathrm{mg} / \mathrm{l}$, to $0.1912 \mathrm{mg} / \mathrm{l}$ (Table 3). Higher values of silicates during summer months may be due to release of silica during decomposition of organic matter at high temperature.

In the present study, phytoplankton comprised five major groups, namely myxophyceae (Blue green algae), Chlorophyceae (Green algae), Desmidiace (Desmids), Bacillariophyceae (Diatoms) and Euglenophyceae (Euglenoids). The Oder of abundance in Wetland A was found to be Myxophyceae> Bacillariophyceae > Chlorophyceae> Euglinophyceae > Desmidiace and in Wetland B it was Chlorophyceae>Myxophyceae> Bacillariophyceae > Euglinopphyceae > Desmidiaceae (Figures1-2). The phytoplankton which could be identified includes 22 genera in Wetland A and 27 genera in Wetland B (Tables 4-5). In Wetland A phytoplankton varied from $51 \mathrm{No} / \mathrm{ml}$ (November, 2001) to $122 \mathrm{No} / \mathrm{ml}$ (April, 2001) while in Wetland B total number varied from $133 \mathrm{No} / \mathrm{ml}$ (July, 2001) to 213No/ml in (September, 2000) and in (Tables 4-5).

Statistically phytoplankton showed significant negative correlation in Wetland A $(r=-0.539)$ only while with nitrate and Phosphate positive in significant correlation in both the ponds. With Silica correlation was found insignificant in both ponds while positive significant with zooplankton, in Wetland B only. Statistically no significant correlation was observed with Gross primary productivity, Net primary and Chlorophyll a. statistically non significant relationship could be due to entry of uncontrolled varied quantum of sewage into these wetlands.

In all the selected ponds Microcystis was found to be the most dominant species among myxophyceae followed by Anabaena species. However, Nostoc was only found in Wetland A. According to the Palmer (1969), the occurrence of Anabaena, Nostoc and Microcystis is the indication of organic pollution and eutrophication.

Among Cholorophyceae Protococus colonies were the more abundantly found in Wetland B (Tables 45). Among Bacillariophyceae Cyclotella, Amphora and Diatoma mostly occur in Wetland B and showed their irregular presence at Wetland A.

The Euglenophyceae group was represented by only two genera, namely Euglena sp. and Phacus sp. were found in quite good numbers, reflecting the idea that they may be highly resistant to the changing environmental conditions and moderate supply of nutrients for their growth and development (Tables 4-5).

In the present study, zooplankton abundance in Wetland A was found to be Cladocera > Copepoda > Rotifera and in Wetland B it was Cladocera >Copepoda > Rotifera > Ostracoda (Tables 6-7).

The group cladocera is represented by Daphnia pulex, Daphnia parvula, Daphanosoma sp., Moina sp. Ceriodaphnia sp, Alonella sp., Leptodora sp., Simocephalus sp and Bosmina sp. Highest Cladocera density was recorded in Wetland B followed by Wetland A (Tables 6-7; Figures 3-4).

The Copepoda represented by Cyclops sp., Canthocamptus sp., Diaptomus sp.,and Limnocalanus sp. Among all Cyclops sp. dominated followed by Diaptomus $s p$. in both the ponds. Limnocalanus $s p$., was only recorded in Wetland A. Later on after one and half decades Ahmad (2016) has also reported Diaptomus sp. in abundance throughout the period of investigations in Macrophyte infested waterbodies.

In the present study, Ostracods were only found and represented by cypris sp., in Wetland B. Different developmental stages of zooplankton were counted together as nauplii and eggs (Tables 6-7).

Primary production includes gross primary productivity (G.P.P), the rate of transformation of radiant energy to chemical, the total production (i.e. production as well respiration), net primary productivity (N.P.P.), the net production left after expenditure in respiration and community respiration (C.R.), the rate of loss of fixed energy in respiration. The values of N.P.P., G.P.P, Community respiration (C.R.) and Chlorophyll ' $a$ ' pigment for both ponds are given in Table 9.

It is clear from the table, the values of gross primary production were always found higher than the values of net primary production. The variations in the rates of production as noted might be due to favourable and unfavourable physico chemical condition during the different months. Higher rates indicate that these water bodies are primarily rich in nutrients with enough lighted zone and energy content. 
Statistically, G.P.P and N.P.P values were found to have a significant positive relationship with Chlorophyll ' $\mathrm{a}$ ' and with water temperature in both the water bodies but with phytoplankton, it showed a non significant correlation in both Wetlands (Table 8).

Community respiration did not show any relationship with plank tonic organisms in both the ponds. It may be because of high rate of decomposition of organic matter in the water bodies and some turbid conditions during different months.

The high values of chlorophyll 'a' were recorded when transparency values was low and vice versa. The high values of Productivity (G.P.P. and N.P.P.) were obtained at the time of high concentration of Chlorophyll 'a' and vice-versa (Table 9).

Table -1. Monthly variations in Air temperature, Water temperature, $\mathrm{pH}$ and Transparency in selected Wetlands

\begin{tabular}{|l|l|l|l|l|l|l|l|l|}
\hline \multirow{2}{*}{ Months } & \multicolumn{2}{|l|}{ Air temp $\left({ }^{0} \mathbf{C}\right)$} & \multicolumn{2}{|l|}{ Water temp $\left(^{\mathbf{0}} \mathbf{C}\right)$} & pH & \multicolumn{2}{l|}{ Transparency (cm) } \\
\cline { 2 - 9 } & W A & W B & W A & W B & W A & W B & W A & W B \\
\hline Aug.2000 & 32.0 & 30.0 & 30.0 & 29.0 & 9.2 & 8.4 & 15.2 & 17.3 \\
\hline Sep. & 30.0 & 30.0 & 28.0 & 28.0 & 9.1 & 8.4 & 17.2 & 17.2 \\
\hline Oct. & 28.0 & 29.0 & 26.0 & 28.0 & 8.8 & 8.4 & 21.1 & 18.3 \\
\hline Nov. & 28.0 & 28.0 & 25.0 & 27.0 & 8.8 & 8.4 & 33.2 & 16.3 \\
\hline Dec. & 19.0 & 22.0 & 17.0 & 21.5 & 8.9 & 8.5 & 28.2 & 28.2 \\
\hline Jan.2001 & 15.0 & 18.0 & 20.0 & 17.0 & 8.8 & 9.1 & 13.1 & 28.3 \\
\hline Feb & 18.0 & 20.0 & 20.0 & 19.0 & 8.7 & 9.0 & 13.5 & 38.3 \\
\hline Mar. & 21.0 & 23.0 & 22.0 & 21.0 & 8.4 & 8.7 & 15.5 & 35.4 \\
\hline Apr. & 23.0 & 25.0 & 23.0 & 17.0 & 8.7 & 8.7 & 14.2 & 26.5 \\
\hline May & 26.0 & 33.0 & 30.0 & 19.0 & 8.6 & 9.2 & 26.2 & 13.5 \\
\hline Jun. & 32.0 & 33.0 & 32.0 & 21.0 & 8.7 & 9.1 & 32.2 & 9.1 \\
\hline July & 36.0 & 32.0 & 32.0 & 23.0 & 8.9 & 9.1 & 35.3 & 14.5 \\
\hline Aug. & 32.0 & 32.0 & 32.0 & 29.0 & 8.3 & 9.1 & 36.5 & 13.5 \\
\hline Sep. & 36.0 & 32.0 & 30.0 & 30.0 & 9.3 & 8.4 & 38.1 & 22.5 \\
\hline Oct. & 31.0 & 32.0 & 25.0 & 28.0 & 9.1 & 8.4 & 29.5 & 25.3 \\
\hline Nov. & 19.0 & 30.0 & 18.0 & 17.0 & 8.8 & 8.4 & 34.5 & 25.5 \\
\hline Dec. & 19.0 & 20.0 & 18.5 & 15.0 & 8.6 & 8.6 & 17.5 & 30.5 \\
\hline
\end{tabular}

Table -2. Monthly variations in DO, TDS, Hardness and Chloride in selected Wetlands

\begin{tabular}{|l|l|l|l|l|l|l|l|l|}
\hline \multirow{2}{*}{ Months } & DO $(\mathbf{m g} / \mathbf{l})$ & \multicolumn{2}{l|}{ TDS (mg/l) } & \multicolumn{2}{l|}{ Hardness (mg/l) } & \multicolumn{2}{l|}{ Chloride (mg/l) } \\
\cline { 2 - 9 } & WA & W B & WA & W B & WA & W B & WA & W B \\
\hline Aug.2000 & 6.2 & 8.0 & 1980.0 & 1260.0 & 240.0 & 142.0 & 52.00 & 56.00 \\
\hline Sep. & 4.2 & 9.3 & 1925.0 & 820.0 & 242.0 & 147.0 & 50.00 & 57.00 \\
\hline Oct. & 4.8 & 6.4 & 1850.0 & 685.0 & 272.0 & 186.0 & 50.00 & 76.00 \\
\hline Nov. & 5.4 & 6.2 & 1300.0 & 1120.0 & 284.0 & 196.0 & 65.00 & 67.00 \\
\hline Dec. & 6.2 & 6.4 & 1910.0 & 1017.0 & 376.0 & 188.0 & 52.00 & 83.00 \\
\hline Jan.2001 & 6.4 & 6.9 & 2490.0 & 1180.0 & 356.0 & 296.0 & 56.00 & 84.00 \\
\hline Feb & 6.2 & 4.0 & 2515.0 & 800.0 & 334.0 & 276.0 & 61.00 & 125.00 \\
\hline Mar. & 9.4 & 5.0 & 2800.0 & 600.0 & 366.0 & 320.0 & 157.00 & 107.00 \\
\hline Apr. & 5.0 & 6.4 & 5600.0 & 1200.0 & 354.0 & 292.0 & 136.00 & 168.00 \\
\hline May & 5.6 & 8.0 & 1350.0 & 2170.0 & 376.0 & 312.0 & 136.00 & 78.00 \\
\hline Jun. & 5.0 & 9.6 & 2400.0 & 1733.0 & 390.0 & 244.0 & 318.00 & 423.00 \\
\hline July & 4.4 & 7.0 & 3000.0 & 1000.0 & 218.0 & 162.0 & 263.00 & 288.00 \\
\hline Aug. & 4.2 & 6.8 & 2120.0 & 1500.0 & 244.0 & 124.0 & 149.00 & 138.00 \\
\hline Sep. & 5.2 & 8.8 & 2010.0 & 720.0 & 294.0 & 188.0 & 170.00 & 153.00 \\
\hline Oct. & 6.4 & 4.4 & 2000.0 & 1069.0 & 374.0 & 264.0 & 199.00 & 170.00 \\
\hline Nov. & 8.0 & 6.0 & 1700.0 & 1170.0 & 390.0 & 260.0 & 214.00 & 306.00 \\
\hline Dec. & 8.2 & 7.0 & 1950.0 & 1060.0 & 380.0 & 275.0 & 213.00 & 318.00 \\
\hline
\end{tabular}


Table -3. Monthly variations in Sulphate, Nitrate, Phosphate and Silica in selected Wetlands

\begin{tabular}{|l|l|l|l|l|l|l|l|l|}
\hline \multirow{2}{*}{ Months } & \multicolumn{2}{l|}{ Sulphate (mg/l) } & \multicolumn{2}{l|}{ Nitrate (mg/l) } & \multicolumn{2}{l|}{ Shosphate (mg/l) $(\mathrm{mg} / \mathrm{l})$} \\
\cline { 2 - 9 } & W A & W B & W A & W B & W A & W B & W A & W B \\
\hline Aug.2000 & 51.00 & 33.00 & 0.157 & 0.117 & 0.586 & 0.635 & 0.0450 & 0.0950 \\
\hline Sep. & 40.00 & 34.00 & 0.081 & 0.087 & 1.040 & 0.785 & 0.0387 & 0.1887 \\
\hline Oct. & 57.00 & 32.00 & 0.081 & 0.112 & 0.586 & 0.541 & 0.0450 & 0.0825 \\
\hline Nov. & 48.00 & 32.00 & 0.134 & 0.056 & 0.586 & 0.570 & 0.0460 & 0.0887 \\
\hline Dec. & 43.00 & 32.00 & 0.182 & 0.052 & 0.419 & 0.707 & 0.1031 & 0.0825 \\
\hline Jan.2001 & 57.00 & 38.00 & 0.122 & 0.092 & 0.620 & 0.550 & 0.1587 & 0.0137 \\
\hline Feb & 87.50 & 82.00 & 0.146 & 0.071 & 0.695 & 0.695 & 0.0225 & 0.0150 \\
\hline Mar. & 153.00 & 42.00 & 0.117 & 0.156 & 0.761 & 0.761 & 0.0218 & 0.0825 \\
\hline Apr. & 164.00 & 55.00 & 0.123 & 0.117 & 0.812 & 0.965 & 0.1912 & 0.0425 \\
\hline May & 153.00 & 104.00 & 0.161 & 0.086 & 1.425 & 0.941 & 0.1862 & 0.0475 \\
\hline Jun. & 142.00 & 179.00 & 0.151 & 0.081 & 0.867 & 1.090 & 0.1887 & 0.1887 \\
\hline July & 100.00 & 57.00 & 0.195 & 0.161 & 0.470 & 0.586 & 0.0975 & 0.0925 \\
\hline Aug. & 88.00 & 51.00 & 0.135 & 0.131 & 0.359 & 0.321 & 0.0725 & 0.1350 \\
\hline Sep. & 97.00 & 34.00 & 0.267 & 0.175 & 0.390 & 0.234 & 0.0837 & 0.0762 \\
\hline Oct. & 100.00 & 49.00 & 0.179 & 0.170 & 0.490 & 0.290 & 0.1662 & 0.1187 \\
\hline Nov. & 108.00 & 53.00 & 0.278 & 0.240 & 0.460 & 0.226 & 0.1775 & 0.1012 \\
\hline Dec. & 135.00 & 43.00 & 0.176 & 0.086 & 0.656 & 0.191 & 0.1887 & 0.0637 \\
\hline
\end{tabular}

Table-4. Monthly abundance and distribution of Phytoplankton (No/ml) in Wetland A

\begin{tabular}{|c|c|c|c|c|c|c|c|c|c|c|c|c|c|c|c|c|c|}
\hline Grups & $\begin{array}{l}\mathrm{AI} \\
\mathrm{g} . \\
00\end{array}$ & Sep & Oct & Sor & $D e$ & Jall & $\mathrm{Feb}$ & Ihr & Ipr & Nay & Jall & $\begin{array}{l}\mathrm{h} \\
1\end{array}$ & tug & Sep & Oet & Yot & Dex \\
\hline \multicolumn{18}{|l|}{ Norwotinoegas } \\
\hline Jifroçsyis sp. & 4 & 3 & 4 & 2 & 3 & 7 & 5 & 8 & 6 & 5 & 10 & 3 & 5 & 4 & 5 & 3 & 3 \\
\hline Ansboensy & 12 & 10 & 12 & 4 & 5 & 4 & 5 & 9 & 6 & 6 & 5 & 13 & 16 & 11 & 13 & 5 & 5 \\
\hline Oxilistorings. & 1 & 0 & 0 & 1 & 0 & 4 & $?$ & 5 & 7 & 1 & 3 & 1 & 1 & 1 & $\overline{0}$ & 0 & 0 \\
\hline Spiolinus. & 4 & 3 & 3 & 4 & 2 & 5 & 3 & j & 9 & 3 & 8 & 5 & 4 & 4 & 5 & 3 & 2 \\
\hline Notorses. & 2 & 4 & 2 & 4 & 4 & 3 & 4 & 3 & 4 & 4 & 7 & 2 & 3 & $j$ & 3 & 5 & 5 \\
\hline Iotal & 23 & 20 & 21 & 15 & 14 & 23 & 10 & 30 & 32 & 19 & 33 & It & 29 & 25 & 86 & 16 & 15 \\
\hline \multicolumn{18}{|l|}{ Coolutuphreace } \\
\hline$\overline{\text { Scandentess }}$ & 2 & 2 & 3 & 4 & 2 & 5 & 3 & 5 & 5 & II & 7 & 4 & 3 & 3 & 4 & 5 & 3 \\
\hline Codlshnueg & 3 & 3 & 4 & 0 & 5 & 6 & $j$ & 5 & 6 & 9 & 8 & 3 & 4 & 4 & 5 & 0 & 6 \\
\hline spiogensple. & 2 & 0 & $?$ & 3 & 0 & 3 & 3 & 4 & 5 & 6 & 4 & 3 & 3 & 0 & 3 & 4 & 0 \\
\hline 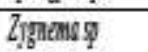 & 2 & 2 & 2 & 0 & 2 & 3 & 2 & 6 & 6 & 2 & 3 & 2 & 3 & 3 & 3 & 0 & 3 \\
\hline 3firosporos s. & 1 & 1 & 1 & 2 & 3 & 4 & 1 & 3 & 3 & 5 & 6 & $?$ & 2 & 2 & 2 & 3 & 4 \\
\hline Selaudnumg. & 3 & 2 & 3 & 2 & 2 & 5 & 4 & 7 & 10 & 5 & 5 & 5 & 4 & 3 & 4 & 3 & 3 \\
\hline Total & 13 & 10 & 15 & 11 & 14 & 26 & 18 & 30 & 35 & 30 & 33 & 19 & 19 & 15 & 21 & 15 & 19 \\
\hline \multicolumn{18}{|l|}{ Deridinene } \\
\hline Closteriants. & 2 & 1 & 2 & 1 & 2 & 2 & 2 & ? & 2 & 1 & 2 & 1 & 2 & 1 & 2 & 0 & 1 \\
\hline Cosmeriamp & 2 & 2 & t & 2 & 3 & 4 & 5 & 3 & 2 & 3 & 2 & 1 & 2 & 1 & 0 & 0 & 1 \\
\hline Deswinineg & 1 & 1 & I & 2 & 1 & 2 & $t$ & 1 & 2 & 2 & 1 & 0 & 1 & 1 & 0 & 2 & 2 \\
\hline Ionl & j & 4 & 4 & 5 & 6 & 8 & 8 & 6 & 6 & 6 & 5 & 2 & 5 & 3 & 2 & 2 & 4 \\
\hline \multicolumn{18}{|l|}{ Barillanophroase } \\
\hline Naticalsp. & 4 & $?$ & 5 & 4 & 3 & 6 & 9 & 11 & 9 & 7 & 5 & 5 & 5 & 3 & 6 & 5 & 3 \\
\hline Nincting & 3 & 4 & 6 & 1 & 1 & 4 & 7 & 7 & 9 & 4 & 4 & 3 & 4 & 5 & $?$ & 0 & 2 \\
\hline Șinetros & 4 & 3 & 4 & 3 & 3 & 7 & 6 & 8 & 12 & 8 & 6 & 4 & 5 & 4 & 5 & 4 & 4 \\
\hline Gidotillogs. & 2 & 1 & 2 & 2 & 2 & 3 & ? & 4 & 5 & 4 & 4 & 1 & 3 & 0 & 3 & 3 & 5 \\
\hline Diatomasp. & 1 & 1 & 0 & 0 & 1 & 3 & 4 & 2 & 2 & 3 & 2 & 2 & 2 & 2 & 2 & 0 & 0 \\
\hline Iotal & it & 11 & 17 & 10 & 10 & 23 & 28 & 32 & 37 & 86 & 21 & 15 & 19 & 40 & 3 & 12 & 14 \\
\hline \multicolumn{18}{|l|}{ Englewothurease: } \\
\hline Eugleansp. & 8 & 10 & 6 & 8 & 10 & 8 & 6 & 4 & 8 & 6 & 4 & 2 & 8 & 6 & 4 & 4 & 6 \\
\hline Phocarg. & 4 & 5 & 6 & 4 & 5 & 4 & 3 & ? & 4 & 3 & 2 & 1 & 4 & 3 & 2 & 2 & 3 \\
\hline Tonl & 12 & 15 & 12 & 12 & 15 & 12 & 9 & 6 & 12 & 9 & 6 & 3 & 12 & 9 & 6 & 6 & 9 \\
\hline $\mathrm{GI}$ & 67 & 60 & 69 & 53 & 59 & 92 & 82 & 124 & 122 & 98 & g8 & 63 & 84 & 66 & 78 & $5 t$ & 61 \\
\hline
\end{tabular}


Table- 5. Monthly abundance and distribution of Phytoplankton $(\mathrm{No} / \mathrm{ml})$ in Wetland B

\begin{tabular}{|c|c|c|c|c|c|c|c|c|c|c|c|c|c|c|c|c|c|}
\hline Groups & $\begin{array}{l}\text { Aug. } \\
2000\end{array}$ & Sep & Oct & Nov & Dec & Jan 01 & Feb & Mar & Apr & May & Jun & Jul & Aug & Sep & Oct & Nov & Dec \\
\hline \multicolumn{18}{|l|}{ Myxophyceae } \\
\hline Microcystis sp. & 62 & 66 & 47 & 32 & 16 & 15 & 32 & 48 & 62 & 62 & 54 & 50 & 60 & 62 & 46 & 30 & 14 \\
\hline Anabaena sp & 4 & 5 & 6 & 4 & 3 & 3 & 6 & 3 & 5 & 7 & 4 & 3 & 3 & 4 & 5 & 3 & 2 \\
\hline Oscillatoria sp. & 1 & 2 & 2 & 3 & 4 & 3 & 3 & 3 & 2 & 6 & 4 & 2 & 1 & 1 & 1 & 2 & 3 \\
\hline Spirullina sp. & 1 & 0 & 0 & 1 & 0 & 3 & 4 & 2 & 4 & 4 & 3 & 1 & 2 & 0 & 1 & 1 & 1 \\
\hline Total & 68 & 73 & 55 & 40 & 23 & 24 & 45 & 56 & 73 & 79 & 19 & 56 & 66 & 67 & 53 & 36 & 20 \\
\hline \multicolumn{18}{|l|}{ Cholorophyceace } \\
\hline Pediastrum sp & 4 & 3 & 2 & 1 & 4 & 3 & 2 & 5 & 4 & 3 & 2 & 1 & 3 & 2 & 1 & 0 & 3 \\
\hline Crucigenia sp & 9 & 11 & 12 & 9 & 7 & 7 & 10 & 11 & 13 & 16 & 12 & 8 & 7 & 10 & 11 & 8 & 6 \\
\hline Ankistrodesmus sp & 8 & 8 & 3 & 14 & 11 & 13 & 11 & 12 & 11 & 13 & 8 & 7 & 7 & 7 & 2 & 12 & 10 \\
\hline Scenedesmus sp. & 16 & 11 & 8 & 16 & 6 & 7 & 22 & 6 & 9 & 6 & 4 & 5 & 12 & 10 & 7 & 12 & 5 \\
\hline Protococcus sp & 14 & 12 & 16 & 18 & 13 & 14 & 15 & 11 & 17 & 18 & 14 & 12 & 12 & 15 & 17 & 12 & 13 \\
\hline Coelastrum sp. & 2 & 2 & 1 & 2 & 1 & 1 & 2 & 3 & 3 & 2 & 3 & 1 & 1 & 1 & 0 & 1 & 0 \\
\hline Chlorellasp & 2 & 2 & 0 & 2 & 0 & 0 & 2 & 3 & 3 & 2 & 3 & 2 & 0 & 1 & 1 & 0 & 1 \\
\hline Tetraspora sp & 1 & 4 & 3 & 2 & 5 & 1 & 0 & 0 & 11 & 1 & 0 & 1 & 0 & 3 & 2 & 1 & 4 \\
\hline Spirogyra sp. & 4 & 1 & 4 & 5 & 3 & 2 & 3 & 3 & 1 & 1 & 2 & 1 & 3 & 0 & 3 & 4 & 2 \\
\hline Ulothrix sp & 3 & 3 & 2 & 4 & 3 & 1 & 0 & 3 & 1 & 0 & 0 & 1 & 2 & 2 & 1 & 3 & 2 \\
\hline Zygnemasp & 3 & 3 & 2 & 2 & 0 & 0 & 0 & 2 & 0 & 1 & 1 & 0 & 0 & 2 & 1 & 1 & 1 \\
\hline Microspora sp. & 2 & 2 & 2 & 1 & 2 & 2 & 0 & 0 & 0 & 1 & 1 & 0 & 1 & 1 & 2 & 1 & 2 \\
\hline Total & 68 & 62 & 55 & 76 & 55 & 51 & 67 & 59 & 73 & 64 & 50 & 39 & 48 & 54 & 48 & 55 & 49 \\
\hline \multicolumn{18}{|l|}{ Desmidiaceae } \\
\hline Closterium sp. & 7 & 5 & 4 & 3 & 5 & 4 & 3 & 3 & 2 & 5 & 5 & 6 & 4 & 7 & 3 & 4 & 4 \\
\hline Cosmarium sp. & 3 & 2 & 4 & 2 & 3 & 2 & 3 & 4 & 2 & 5 & 4 & 3 & 2 & 1 & 3 & 1 & 2 \\
\hline Genicularia sp. & 2 & 2 & 3 & 3 & 1 & 1 & 4 & 3 & 2 & 2 & 1 & 0 & 1 & 1 & 2 & 2 & 0 \\
\hline Total & 12 & 9 & 11 & 8 & 9 & 7 & 10 & 10 & 6 & 12 & 10 & 9 & 7 & 9 & 8 & 7 & 6 \\
\hline \multicolumn{18}{|l|}{ Bacillariophyceace } \\
\hline Navicula sp. & 11 & 14 & 16 & 5 & 12 & 17 & 13 & 4 & 15 & 6 & 3 & 4 & 10 & 12 & 15 & 4 & 10 \\
\hline Nitzchia sp. & 12 & 13 & 14 & 4 & 7 & 18 & 8 & 3 & 6 & 7 & 4 & 7 & 11 & 12 & 13 & 3 & 6 \\
\hline Synedra sp. & 5 & 8 & 9 & 11 & 8 & 9 & 7 & 4 & 6 & 5 & 4 & 3 & 4 & 7 & 8 & 10 & 7 \\
\hline Cyclotella sp. & 3 & 4 & 3 & 2 & 4 & 3 & 4 & 2 & 3 & 4 & 2 & 1 & 2 & 3 & 2 & 1 & 3 \\
\hline Amphora & 3 & 4 & 4 & 4 & 6 & 4 & 4 & 3 & 2 & 3 & 2 & 1 & 2 & 3 & 3 & 2 & 5 \\
\hline Diatoma sp. & 2 & 3 & 3 & 3 & 4 & 3 & 4 & 2 & 2 & 5 & 3 & 2 & 1 & 2 & 2 & 1 & 3 \\
\hline Total & 36 & 46 & 49 & 29 & 41 & 54 & 40 & 18 & 34 & 30 & 18 & 18 & 30 & 39 & 43 & 21 & 34 \\
\hline \multicolumn{18}{|l|}{ Euglenophyceace } \\
\hline Euglena sp. & 8 & 8 & 10 & 10 & 19 & 19 & 9 & 10 & 14 & 5 & 3 & 8 & 7 & 6 & 9 & 10 & 18 \\
\hline Phacus sp. & 10 & 15 & 16 & 17 & 15 & 15 & 8 & 7 & 10 & 8 & 5 & 3 & 9 & 12 & 15 & 16 & 13 \\
\hline Total & 18 & 23 & 12 & 27 & 34 & 34 & 17 & 17 & 24 & 13 & 8 & 13 & 16 & 18 & 24 & 26 & 31 \\
\hline GT & 202 & 213 & 196 & 180 & 162 & 170 & 179 & 160 & 210 & 198 & 148 & 133 & 167 & 187 & 176 & 145 & 140 \\
\hline
\end{tabular}

Table-6. Monthly abundance and distribution of Zooplankton (No. /L) in Wetland A

\begin{tabular}{|c|c|c|c|c|c|c|c|c|c|c|c|c|c|c|c|c|c|}
\hline Genera & Aug00 & Sep & Oct & Nov & Dec & $\operatorname{Tan} 01$ & Feb & $\mathrm{Mar}$ & Apr & May & $\mathrm{Jun}$ & hy & Ang & sep & Oct & Nov & Dec \\
\hline \multicolumn{18}{|l|}{ Rocifers } \\
\hline Brachionusculciflorus & 6 & 10 & 2 & 4 & 8 & 6 & 7 & 6 & 2 & 4 & 9 & 6 & 5 & 7 & 5 & 4 & 3 \\
\hline Rotaria sp & 2 & 3 & 4 & 12 & 1 & 1 & 0 & 0 & 0 & 1 & 2 & 1 & 0 & 2 & 3 & 6 & 1 \\
\hline Eenutella sp & 0 & 4 & 2 & 1 & 6 & 2 & 1 & 0 & 0 & 1 & 0 & 0 & 0 & 2 & 3 & 8. & 4 \\
\hline Firuia sp & 10 & 4 & 5 & 3 & 5 & 4 & 5 & 3 & 5 & 7 & 12 & 16 & 25 & 28 & 18 & 16 & 8 \\
\hline Notholcusp & 4 & 3 & 3 & 2 & 1 & 0 & 1 & 1 & 0 & 1 & 1 & 1 & 3 & 2 & 1 & 2 & 1 \\
\hline Total & 22 & 24 & 16 & 22 & 21 & 13 & 14 & 12 & 9 & 19 & 28 & 33 & 36 & 31 & 28 & 28 & 13 \\
\hline \multicolumn{18}{|l|}{ Cadocerz } \\
\hline Daphoin sp & 12 & 10 & 15 & 13 & 13 & 10 & 8 & 20 & 25 & 15 & 10 & 14 & 18 & 22 & 23 & 25 & 21 \\
\hline Ceriodaphnias & 1 & 1 & 0 & 0 & 0 & 0 & 0 & 0 & 0 & 0 & 0 & 0 & 3 & 5 & 0 & 0 & 0 \\
\hline Moine sp & 3 & 2 & 4 & 2 & 3 & 0 & 5 & 5 & 7 & 3 & 2 & 4 & 2 & 5 & 9 & 7 & 2 \\
\hline Simocephalus sp & 6 & 7 & 8 & 8 & 9 & 4 & 5 & 6 & 7 & 4 & 5 & 4 & 6 & 6 & 7 & 7 & 8 \\
\hline Bosmina sp & 1 & 2 & 1 & 0 & 0 & 0 & 1 & 0 & 0 & 0 & 0 & 0 & 0 & 3 & 4 & 2 & 1 \\
\hline Total & 23 & 22 & 28 & 23 & 25 & 14 & 19 & 31 & 39 & 22 & 17 & 22 & 32 & 42 & 45 & 40 & 32 \\
\hline \multicolumn{18}{|l|}{ Copepoda } \\
\hline Cyclops sp & 15 & 18 & 13 & 10 & 8 & 3 & 4 & 11 & 9 & 11 & 17 & 22 & 18 & 22 & 11 & 12 & 11 \\
\hline Conthocampus sp & 0 & 0 & 0 & 0 & 0 & 0 & 2 & 0 & 1 & 0 & 2 & 5 & 0 & 0 & 0 & 1 & 0 \\
\hline Dimptomus sp & 7 & 13 & 5 & 6 & 4 & 8 & 4 & 6 & 8 & 7 & 13 & 15 & 14 & 15 & 10 & 2 & 3 \\
\hline Limnocalonussp & 3 & 4 & 2 & 2 & 3 & 2 & 4 & 2 & 3 & 1 & 8 & 13 & 4 & 3 & 4 & 1 & 2 \\
\hline \multicolumn{18}{|l|}{ Total } \\
\hline Eggs & 5 & 4 & 5 & 3 & 8 & 5 & 2 & 3 & 2 & 4 & 6 & 6 & 10 & 4 & 4 & 3 & 2 \\
\hline Nasplin & 5 & 6 & 13 & 12 & 8 & 6 & 7 & 3 & 4 & 5 & 6 & 7 & 11 & 15 & 10 & 6 & 5 \\
\hline Grand total & 80 & 91 & 82 & 78 & 77 & 51 & 56 & 68 & 75 & 72 & 97 & 123 & 125 & 133 & 112 & 94 & 69 \\
\hline
\end{tabular}


Table-7. Monthly abundance and distribution of Zooplankton (No./L) in Wetland B

\begin{tabular}{|c|c|c|c|c|c|c|c|c|c|c|c|c|c|c|c|c|c|}
\hline Genesa & $A=00$ & Sep. & Oct & Nay & Dec & $\operatorname{Jan} 01$ & Feb. & Mar & Apt & May & Jun & July & Aulg & Sep. & adt & Nov & Dec \\
\hline \multicolumn{18}{|l|}{ Rotifers } \\
\hline Brachionus angularì & 4 & 4 & 7 & 5 & 3 & $\mathbf{3}$ & 2 & 2 & 3 & 4 & 4 & 2 & 4 & 3 & 6 & 4 & 5 \\
\hline Brachionuscalctionus & 4 & 6 & 7 & 5 & 3 & 3 & 2 & 2 & 3 & 4 & 4 & 2 & 3 & 5 & 6 & 5 & 3 \\
\hline Keralella s tropica & 2 & 1 & 1 & 2 & 2 & 1 & 2 & 1 & 2 & 0 & 0 & 1 & 1 & 1 & 2 & 2 & 2 \\
\hline Filinia sp & 5 & 4 & 3 & 2 & 3 & 2 & 3 & 3 & 3 & 2 & 6 & 5 & 4 & 3 & 2 & 2 & 3 \\
\hline Testudinella sp & 1 & 1 & 0 & 0 & $\theta$ & 0 & 0 & 0 & 0 & 0 & 0 & 0 & 0 & 0 & 1 & 0 & 0 \\
\hline Asplanches sp & 1 & 0 & 1 & 1 & 2 & 2 & 1 & 2 & 1 & 1 & 0 & 0 & 2 & $t$ & 0 & 2 & 2 \\
\hline Lecant sp & 0 & 0 & 0 & 0 & 0 & 0 & 0 & 0 & 0 & 0 & 0 & 0 & 0 & 0 & 0 & 0 & 1 \\
\hline Nothales sp & 2 & 3 & 2 & 1 & 0 & 0 & 3 & 1 & 3 & 2 & 1 & 3 & 2 & 3 & 2 & 2 & 1 \\
\hline Total & 19 & 19 & 21 & 16 & 13 & 11 & 13 & 11 & 15 & 13 & 15 & 13 & 16 & 16 & 20 & 17 & 17 \\
\hline \multicolumn{18}{|l|}{ Cladocers } \\
\hline Dephinia piakx & 9 & 10 & 16 & 13 & 13 & 16 & 15 & 12 & 16 & 9 & 6 & 5 & 9 & II & 15 & 12 & 12 \\
\hline Daphnia parvela & 2 & 6 & 5 & 7 & 3 & 4 & 1 & 1 & 1 & 1 & 0 & 0 & 2 & 4 & 3 & 6 & 3 \\
\hline Daphnia magna & 11 & 16 & 13 & 13 & 16 & 15 & 11 & 16 & 9 & 6 & 5 & 8 & 10 & 12 & 11 & 12 & 11 \\
\hline Daphnia carinata & 2 & 4 & 5 & 7 & 3 & 3 & 1 & 1 & 2 & 1 & 2 & $\mathrm{t}$ & 0 & $\mathrm{t}$ & 2 & 4 & 3 \\
\hline Ceriodaphenia & 4 & 3 & 2 & 3 & 3 & 2 & 3 & 2 & 3 & 2 & 2 & 2 & 1 & 2 & 3 & 1 & 2 \\
\hline Soina sp & 5 & 5 & 4 & 5 & 3 & 4 & 4 & 3 & 4 & 2 & $t$ & 1 & 2 & 1 & $\frac{2}{2}$ & 2 & 3 \\
\hline Diaphnosoma & 4 & 5 & 4 & 3 & 5 & 6 & 5 & 6 & 4 & 3 & 2 & 2 & 3 & 4 & 3 & 4 & 5 \\
\hline Alonella sp. & 3 & 4 & 3 & 4 & 4 & 3 & 4 & 3 & 4 & 2 & 3 & t & 0 & 2 & 3 & 2 & 3 \\
\hline Simocephalus ip & 1 & 0 & 2 & 1 & 2 & I & 3 & 2 & 2 & 0 & 0 & 0 & 0 & 0 & 0 & 2 & 1 \\
\hline Lepiodera sp & 2 & 2 & I & 2 & 3 & 1 & 2 & 3 & 2 & 1 & 0 & 0 & 1 & 1 & 0 & 1 & 2 \\
\hline Tetal & 43 & 55 & 51 & 58 & 55 & 49 & 49 & 49 & 47 & 27 & 21 & 20 & 28 & 38 & 42 & 46 & 45 \\
\hline \multicolumn{18}{|l|}{ Copepoda } \\
\hline Crelops sp & II & 12 & 13 & 11 & 9 & 2 & 12 & 12 & 15 & 9 & 6 & 7 & 9 & 10 & 11 & 10 & 8 \\
\hline Canthocampus ip & 4 & 3 & 2 & 2 & 1 & 3 & 4 & 0 & 0 & 1 & 0 & 0 & 3 & 2 & 1 & 1 & 1 \\
\hline Diaptomus sp & 7 & 13 & 9 & $1 \mathrm{t}$ & 8 & 11 & 13 & 9 & 7 & 4 & 3 & 5 & 6 & 12 & B & 10 & 9 \\
\hline \multicolumn{18}{|l|}{ Ostraceda } \\
\hline Cypris sp & 4 & 3 & 2 & 2 & 3 & 1 & 4 & 3 & 2 & 3 & 2 & 2 & 3 & 2 & 1 & 1 & 2 \\
\hline Total & 22 & 28 & 24 & 24 & 24 & 18 & 16 & 29 & 21 & 22 & 14 & 9 & 12 & 25 & 21 & 21 & 18 \\
\hline Eggs & 1 & 2 & 1 & 1 & 2 & $t$ & 2 & 1 & 1 & 3 & 2 & 1 & 0 & 1 & 2 & 1 & 1 \\
\hline Nonplt & 3 & 2 & 1 & 0 & 2 & 3 & 1 & 2 & 3 & 31 & $\frac{1}{1}$ & $t$ & 2 & 1 & 0 & 1 & 1 \\
\hline Graed total & 92 & 109 & 100 & $10 \mathrm{I}$ & 93 & 87 & 98 & 87 & 90 & 61 & 50 & 49 & 65 & 83 & 85 & 87 & 84 \\
\hline
\end{tabular}

Table -8. Statistical brief $(\mathrm{P}<0.05)$ of various water quality parameters in Wetland B and Wetland A

\begin{tabular}{|c|c|c|c|}
\hline Parameters & Parameters & Ponds & Coefficient of Correlation ' $r$ ' \\
\hline \multirow[t]{2}{*}{ Air Temperature } & WaterTemperature & WB & 0.987 \\
\hline & & WA & 0.843 \\
\hline \multirow[t]{10}{*}{ WaterTemperature } & Transparency & WB & -0.737 \\
\hline & & WA & -0.639 \\
\hline & D.O. & WB & 0.453 \\
\hline & & WA & -0.656 \\
\hline & Zooplankton & WB & -0.254 \\
\hline & & WA & 0.240 \\
\hline & Phytoplankton & WB & 0.256 \\
\hline & & WA & -0.539 \\
\hline & Total Dissolved Solids & WB & 0.090 \\
\hline & & WA & 0.116 \\
\hline \multirow[t]{6}{*}{ Dissolved oxygen } & Zooplankton & WB & -0.685 \\
\hline & & WA & -0.623 \\
\hline & Phytoplankton & WB & -0.419 \\
\hline & & WA & 0.538 \\
\hline & Cladocera & WB & 0.411 \\
\hline & & WA & 0.183 \\
\hline \multicolumn{4}{|l|}{$\mathrm{PO}_{4}-\mathrm{P}$} \\
\hline & Copepods & WB & 0.603 \\
\hline & & WA & -0.798 \\
\hline & Ostracoda & WB & -0.631 \\
\hline & & WA & -0.327 \\
\hline & Euglenophycea & WB & -0.577 \\
\hline & & WA & -0.100 \\
\hline & Rotifer & WB & 0.223 \\
\hline & & WA & 0.289 \\
\hline & Mxyophyceae & WB & -0.236 \\
\hline & & WA & -0.534 \\
\hline & Chlorophyceae & WB & -0.459 \\
\hline & & WA & -0.511 \\
\hline & Bacillariophyceae & WB & 0.000 \\
\hline & & WA & -0.495 \\
\hline & Descmidiacea & WB & -0.349 \\
\hline & & WA & -0.592 \\
\hline $\mathrm{NO}_{3}-\mathrm{N}$ & Phytoplankton & WB & -0.798 \\
\hline
\end{tabular}


Limnological status and plankton dynamics in two fresh water wetlands of Aligarh, North India

\begin{tabular}{|c|c|c|c|}
\hline & & WA & -0.357 \\
\hline & Mxyophyceae & WB & -0.631 \\
\hline & & WA & -0.327 \\
\hline & Chlorophyceae & WB & -0.377 \\
\hline & & WA & 0.078 \\
\hline & Bacillariophyceae & WB & 0.446 \\
\hline & & WA & 0.361 \\
\hline & Descmidiacea & WB & 0.330 \\
\hline & & WA & 0.652 \\
\hline & Zooplankton & WB & 0.546 \\
\hline & & WA & 0.224 \\
\hline \multirow[t]{10}{*}{ Chloride } & Mxyophyceae & WB & 0.193 \\
\hline & & WA & 0.474 \\
\hline & Chlorophyceae & WB & 0.364 \\
\hline & & WA & -0.022 \\
\hline & Bacillariophyceae & WB & 0.075 \\
\hline & & WA & 0.150 \\
\hline & Descmidiacea & WB & 0.334 \\
\hline & & WA & -0.504 \\
\hline & Phytoplankton & WB & -0.027 \\
\hline & & WA & 0.491 \\
\hline \multirow[t]{4}{*}{ Gross Primary Productivity } & Chlorophyll a & WB & 0.887 \\
\hline & & WA & 0.536 \\
\hline & Phytoplankton & WB & 0.005 \\
\hline & & WA & -0.138 \\
\hline \multirow[t]{4}{*}{ Net Primary Productivity } & Phytoplankton & WB & 0.044 \\
\hline & & WA & -0.162 \\
\hline & Chlorophyll a & WB & 0.865 \\
\hline & & WA & 0.497 \\
\hline
\end{tabular}

Table -9. Monthly variations in Primary Productivity, Community Respiration and Chlorophyll 'a' in selected Wetlands

\begin{tabular}{|c|c|c|c|c|c|c|c|c|}
\hline \multirow[t]{2}{*}{ Months } & \multicolumn{2}{|c|}{$\begin{array}{l}\text { Net } \\
\text { Productivity g C/m³ } / \mathrm{hr}\end{array}$} & \multicolumn{2}{|c|}{$\begin{array}{l}\text { Gross } \\
\text { productivity g C } / \mathrm{m}^{3} / \mathrm{hr}\end{array}$} & \multicolumn{2}{|c|}{$\begin{array}{l}\text { Community } \\
\text { Respiration g C/m } / \mathrm{m}^{3} / \mathrm{hr}\end{array}$} & \multicolumn{2}{|c|}{$\begin{array}{l}\text { Chlorophyll 'a' } \\
\text { (mg Pigment/L) }\end{array}$} \\
\hline & Wetland A & Wetland B & Wetland A & Wetland B & Wetland A & Wetland B & Wetland A & Wetland B \\
\hline August 00 & 1.437 & 1.628 & 2.115 & 1.717 & 0.089 & 0.678 & 3.317 & 2.452 \\
\hline September & 1.322 & 1.329 & 1.473 & 1.492 & 0.163 & 0.151 & 2.683 & 2.708 \\
\hline October & 1.472 & 1.482 & 1.561 & 1.693 & 0.211 & 0.089 & 3.685 & 3.262 \\
\hline November & 1.622 & 1.632 & 1.734 & 1.952 & 0.320 & 0.112 & 2.945 & 3.252 \\
\hline December & 1.351 & 1.121 & 1.442 & 1.242 & 0.121 & 0.091 & 2.545 & 1.348 \\
\hline January 01 & 0.892 & 0.687 & 1.064 & 0.823 & 0.136 & 0.172 & 2.075 & 0.831 \\
\hline Feburary & 0.564 & 0.923 & 0.675 & 1.023 & 0.100 & 0.111 & 0.786 & 0.562 \\
\hline March & 1.320 & 1.372 & 1.421 & 1.419 & 0.047 & 0.101 & 2.832 & 2.610 \\
\hline April & 1.532 & 1.572 & 1.604 & 1.713 & 0.141 & 0.0072 & 2.715 & 2.920 \\
\hline May & 1.746 & 1.638 & 1.834 & 1.823 & 0.186 & 0.088 & 3.146 & 3.130 \\
\hline June & 1.260 & 1.725 & 1.673 & 2.130 & 0.405 & 0.413 & 3.340 & 3.210 \\
\hline July & 1.836 & 1.523 & 2.240 & 1.813 & 0.290 & 0.404 & 2.351 & 3.070 \\
\hline August & 1.448 & 1.617 & 2.106 & 1.816 & 0.199 & 0.658 & 3.328 & 2.561 \\
\hline September & 1.331 & 1.219 & 1.462 & 1.501 & 0.282 & 0.131 & 2.784 & 2.819 \\
\hline October & 1.482 & 1.391 & 1.572 & 1.581 & 0.190 & 0.090 & $3 . .798$ & 3.151 \\
\hline November & 1.732 & 1.732 & 1.842 & 1.831 & 0.099 & 0.011 & 1.735 & 3.261 \\
\hline December & 1.242 & 1.142 & 1.286 & 1.231 & 0.089 & 0.044 & 2.473 & 1.437 \\
\hline
\end{tabular}

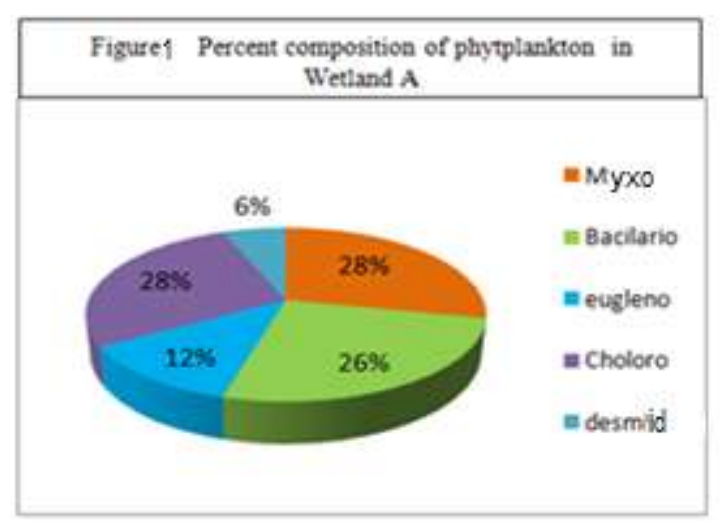

DOI: $10.9790 / 2402-100901120128$

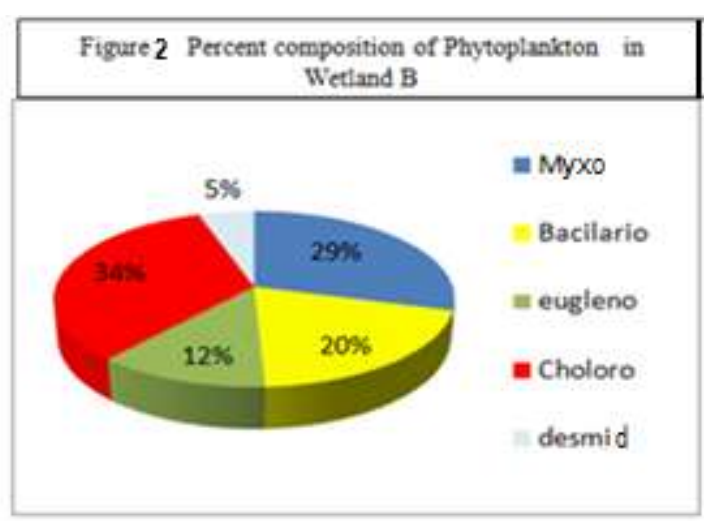

www.iosrjournals.org 

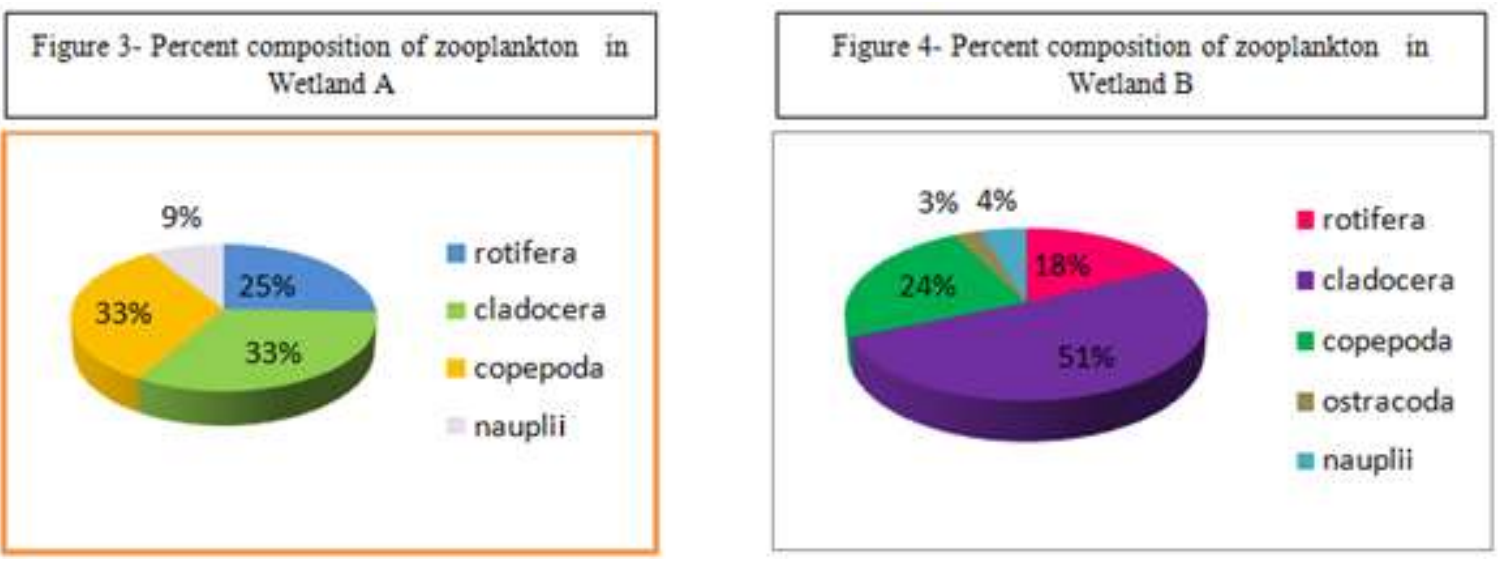

\section{Conclusion}

Study revealed that plankton; both phytoplankton and zooplankton are well represented in these wetlands. The studied wetlands are highly productive showing plankton abundance during summer and post monsoon months. These are primarily rich in nutrients with enough lighted littoral zone and energy content. On the basis of present findings it is concluded that these wetlands being productive in nature can be used for pisciculture or for integrated fish farming.

\section{Acknowledgements}

The first author is grateful to late Professor Asif A. Khan, Ex Chairman and Ex Dean Faculty of Life Sciences, Aligarh Muslim University Aligarh for providing research guidance and laboratory facilities.

\section{References}

[1]. Ahmad U (2016). A study on limnology and biodiversity of crustaceans in macrophyte infested waterbodies of Aligarh region. Thesis Ph.D., Aligarh Muslim University, Aligarh.

[2]. APHA (1998). Standard methods for examination of water and wastewater. American Public Health Association, AWWA, WPCF, Washington, D.C. (U.S.A.), 1193.

[3]. Edmondson, W.T. (1959). Ward and Whipple's Freshwater Biology. (2 ${ }^{\text {nd }}$ Ed). John Wiley \& Sons Inc., New York, 1248 pp.

[4]. Gaarder, T. and Gran, H.H. (1927). Investigation on the primary production of plankton in the Oso Fjord. Rapp. cons. Explor. Mar., 144:56-60.

[5]. Ganapati, S.V. (1960). Ecology of tropical waters. Proc. ICAR Symp. On Algology, New Delhi (1958), $200-218$.

[6]. Nayar, S., Gupta, T.R.C. and Gowda, G. (1999). Secondary production zooplankton biomass in a tropical coastal lagoon near Mangalore, southwest coast of India. Indian J. Fish., 46(3): 281-288.

[7]. Needham, J.G. and Needham, P.R. (1962). A Guide to the Study of the Freshwater Biology. Holden-Dey Inc., Francisco, 108 pp.

[8]. Palmer, C.M. (1969). A composite rating of algae tolerating organic pollution. J. Phycology, 5: 78-82.

[9]. Pennak, R.W. (1978). Freshwater Invertebrates of United States. (2 ${ }^{\text {nd }}$ Ed). John Wiley \& Sons Inc., New York, 803pp.

[10]. Strickland, J.D.H and Pearson, T.R (1972). A Practical Handbook of Sea water Analysis, 2nd Ed.Bull. Res.Bd. Canada,167: 310 pp.

[11]. Theroux, F.R., Eldridge, E.F. and Mallmann, W.L. (1943). Laboratory Manual for Chemical; and Bacterial Analysis of Water and Sewage. McGraw Hill

[12]. Tonapi, G.T. (1980). Freshwater Animals of India: An Ecological Approach. Oxford and IBH Publishing Co., New Delhi, India, 341pp.

[13]. Trivedy, R.K. and Goel, P.K. (1984). Chemical and Biological Methods for Water Pollution Studies. Environmental Publications, Karad, (India), 215pp.

[14]. Vollenweider, R. A. (1969) - Manual of the Methods for Measuring Primary Production in Aquatic Environment. IPB Handbook No. 12, Blackwell Scientific Publications, Oxford, 213

[15]. WWF (1987) - World Wide Fund for Nature for the Ministry of Environment and Forest, Government of India

[16]. WWF (1992) - India's Wetlands Mangrooves and Coral Reefs. WWF- India for the Ministry of Environment and forests, Government of India, New Delhi, India,61 pp. 